\title{
Carfilzomib or bortezomib in combination with cyclophosphamide and dexamethasone followed by carfilzomib maintenance for patients with multiple myeloma after one prior therapy: results from a multicenter, phase II, randomized, controlled trial (MUKfive)
}

Haematologica 2021

Volume 106(10):2694-2706

\section{Correspondence:}

KWEE L YONG

kwee.yong@ucl.ac.uk

Received: January 25, 2021.

Accepted: April 14, 2021.

Pre-published: April 29, 2021.

https://doi.org/10.3324/haematol.2021.278399

(C)2021 Ferrata Storti Foundation

Material published in Haematologica is covered by copyright. All rights are reserved to the Ferrata Storti Foundation. Use of published material is allowed under the following terms and conditions:

https://creativecommons.org/licenses/by-nc/4.0/legalcode. Copies of published material are allowed for personal or internal use. Sharing published material for non-commercial purposes is subject to the following conditions:

https://creativecommons.org/licenses/by-nc/4.0/legalcode, sect. 3. Reproducing and sharing published material for commercial purposes is not allowed without permission in writing from the publisher.
Kwee L. Yong, ${ }^{1 *}$ Samantha Hinsley,,${ }^{2 *}$ Holger W. Auner, ${ }^{3}$ Ceri Bygrave, ${ }^{4}$ Martin F. Kaiser, ${ }^{5}$ Karthik Ramasamy, ${ }^{6}$ Ruth M. de Tute, ${ }^{7}$ Debbie Sherratt, ${ }^{2}$ Louise Flanagan, ${ }^{2}$ Mamta Garg, ${ }^{8}$ Stephen Hawkins, ${ }^{9}$ Catherine Williams, ${ }^{10}$ Jamie Cavenagh, ${ }^{11}$ Neil K. Rabin,${ }^{12}$ James Croft, ${ }^{5}$ Gareth Morgan, ${ }^{13}$ Faith Davies,${ }^{13}$ Roger G. Owen ${ }^{14}$ and Sarah R. Brown ${ }^{2}$

${ }^{1}$ Cancer Institute, University College London, London, UK; ${ }^{2}$ Clinical Trials Research Unit, Leeds Institute of Clinical Trials Research, University of Leeds, Leeds, UK; ${ }^{3}$ Department of Immunology and Inflammation and The Hugh and Josseline Langmuir Centre for Myeloma Research, Imperial College London, London, UK; ${ }^{4}$ Cardiff and Vale University Health Board, Cardiff, UK; ${ }^{5}$ The Institute of Cancer Research and The Royal Marsden Hospital NHS Foundation Trust, London, UK; ${ }^{6}$ Department of Clinical Haematology, Oxford University Hospitals NHS Trust, Oxford, UK; ' Department of Clinical Haematology, Leeds Teaching Hospitals NHS Trust, Leeds, UK; ${ }^{8}$ Department of Haematology, University Hospitals of Leicester NHS Trust, Leicester, UK; ${ }^{9}$ The Clatterbridge Cancer Centre, Liverpool, UK; ${ }^{10} \mathrm{Centre}$ for Clinical Haematology, Nottingham University Hospitals, Nottingham, UK ${ }^{11}$ Department of Haematology, St Bartholomew's Hospital, London, UK; ${ }^{12}$ Department of Haematology, University College Hospital, London, UK; ${ }^{13}$ Perlmutter Cancer Center, NYU Langone Health, New York, NY, USA and ${ }^{14}$ Haematological Malignancy Diagnostic Service (HMDS), St James's University Hospital, Leeds, UK

${ }^{\star} K L Y$ and $S H$ contributed equally as co-first authors.

\section{ABSTRACT}

The proteasome inhibitors, carfilzomib and bortezomib, are widely used to treat myeloma but head-to-head comparisons have produced conflicting results. We compared the activity of these proteasome inhibitors in combination with cyclophosphamide and dexamethasone (KCd vs. VCd) in second-line treatment using fixed duration therapy and evaluated the efficacy of carfilzomib maintenance. MUKfive was a phase II controlled, parallel group trial that randomized patients $(2: 1)$ to $\mathrm{KCd}(\mathrm{n}=201)$ or VCd ( $\mathrm{n}=99)$; responding patients on carfilzomib were randomized to maintenance carfilzomib $(n=69)$ or no further treatment $(n=72)$. Primary endpoints were: (i) very good partial response (non-inferiority, odds ratio [OR] 0.8) at 24 weeks, and (ii) progression-free survival. More participants achieved a very good partial response or better with carfilzomib than with bortezomib (40.2\% vs. $31.9 \%$, OR $=1.48,90 \%$ confidence interval [CI]: 0.95, 2.31; non-inferior), with a trend for particular benefit in patients with adverse-risk disease. $\mathrm{KCd}$ was associated with higher overall response (partial response or better, $84.0 \%$ vs. $68.1 \%$, $\mathrm{OR}=2.72,90 \%$ CI: $1.62,4.55, P=0.001$ ). Neuropathy (grade $\geq 3$ or $\geq 2$ with pain) was more common with bortezomib (19.8\% vs. $1.5 \%, P<0.0001)$, while grade $\geq 3$ cardiac events and hypertension were only reported in the $\mathrm{KCd}$ arm (3.6\% each). The median progression-free survival in the $\mathrm{KCd}$ arm was 11.7 months vs. 10.2 months in the VCd arm (hazard ratio $[\mathrm{HR}]=0.95,80 \% \mathrm{CI}: 0.77,1.18)$. Carfilzomib maintenance was associated with longer progression-free survival, median 11.9 months vs. 5.6 months for no maintenance (HR 0.59, 80\% CI: $0.46-0.77, P=0.0086$ ). When used as fixed duration therapy in first relapase, $\mathrm{KCd}$ is at least as effective as VCd, and carfilzomib is an effective maintenance agent. This trial was registered with International Standard Randomised Controlled Trial Number (ISRCTN) identifier: ISRCTN17354232. 


\section{Introduction}

Proteasome inhibitors form the backbone of many regimens used to treat multiple myeloma (MM). ${ }^{1}$ Bortezomib is a boronic acid-based reversible proteasome inhibitor commonly combined with corticosteroids, alkylating agents, immunomodulatory drugs, or antibodies. ${ }^{2}$ Peripheral neuropathy is a common cause for treatment discontinuation, although this problem may be mitigated by subcutaneous and once-weekly (instead of bi-weekly) administration. ${ }^{3}$ Carfilzomib is an epoxyketone drug that binds irreversibly to the proteasome and is approved for the treatment of relapsed myeloma in combination with dexamethasone, or dexamethasone plus lenalidomide, or daratumumab.4-6 While carfilzomib has limited neurotoxicity, it has been linked to clinically relevant cardiovascular complications, in particular hypertension, heart failure, and renal failure.' Both bortezomib and carfilzomib predominantly target the $\beta 5$ subunit of the constitutive proteasome and the immunoproteasome; at higher concentrations they also co-inhibit either $\beta 1$ and/or $\beta 2$ subunits with different inhibition profiles that determine cytotoxicity and may thus be clinically relevant. ${ }^{8}$

Head-to-head comparison studies of carfilzomib with bortezomib have yielded mixed results. In patients with relapsed or refractory multiple myeloma (RRMM), carfilzomib was compared to bortezomib, both given with dexamethasone: it was found that carfilzomib improved both progression-free survival (PFS) and overall survival (OS). ${ }^{4,9}$ In this study (ENDEAVOR), the carfilzomib dose was $56 \mathrm{mg} / \mathrm{m}^{2}$ twice weekly, and patients received treatment until progression. In contrast, carfilzomib did not improve PFS, OS, or response rates compared to bortezomib when given in combination with melphalan and prednisolone in transplant-ineligible patients with newly diagnosed multiple myeloma (NDMM) ${ }^{10}$ The carfilzomib dose used in this trial (CLARION) was $36 \mathrm{mg} / \mathrm{m}^{2}$ twice weekly, and treatment duration was fixed at nine cycles. Similarly, carfilzomib given at a dose of $36 \mathrm{mg} / \mathrm{m}^{2}$ was not superior to bortezomib when given in combination with lenalidomide and dexamethasone for a fixed period (36 weeks) in standard-risk NDMM patients without intention for immediate autologous stem-cell transplantation (ASCT). ${ }^{11}$ Thus, the optimal choice of proteasome inhibitor, combination, dose and duration of therapy, remains to be established. Superior results seen with carfilzomib in ENDEAVOR indicate a safety profile that lends itself to continuous treatment approaches, suggesting suitability for maintenance. Maintenance with carfilzomib following fixed-duration treatment with cyclophosphamide and dexamethasone in NDMM transplant-ineligible patients has been reported. ${ }^{12}$ The role of carfilzomib maintenance treatment in patients with RRMM remains unexplored.

Triplet regimens have become standard of care for RRMM in patients who are sufficiently fit and these regimens often contain a proteasome inhibitor. The combination of either carfilzomib or bortezomib with dexamethasone and cyclophosphamide is effective $e^{12-14}$ and economically less challenging than three-drug combinations that contain two novel agents. We designed the Myeloma UK five (MUKfive) study, a phase II randomized, controlled, parallel group, multicenter trial for MM patients at first relapse or refractory to just one treatment line, with two main objectives. The first was to compare, in a uniform group of patients, the efficacy of carfilzomib $(\mathrm{K})$ and bortezomib $(\mathrm{V})$, given in combination with cyclophosphamide (C) and dexamethasone (d), i.e., $\mathrm{KCd}$ versus VCd, as fixed-duration therapy (24 weeks), in achieving at least very good partial responses (VGPR). The second objective was to evaluate the PFS benefit of maintenance with single-agent carfilzomib in patients responding to $\mathrm{KCd}$.

\section{Methods}

The full trial protocol, including eligibility criteria and sample size, have already been published. ${ }^{15}$ The trial received national research ethics approval from the NHS National Research Ethics Service London, (REC number: 12/LO/1078). Three hundred participants were randomized $(2: 1)$ to $\mathrm{KCd}$ or $\mathrm{VCd}$, using minimization with a random element. KCd participants in at least stable disease after six cycles were randomized (1:1) to receive maintenance carfilzomib or observation. Figure 1 shows the minimization factors and treatment regimens.

The MUKfive trial had two co-primary endpoints: (i) a comparison of the induction regimens' capacity to produce $\geq \mathrm{VGPR}$ at 24 weeks after initial randomization (non-inferiority [NI] comparison with NI margin $-5 \%$ [difference in proportion] $/ 0.8$ [odds ratio; OR], one-sided $5 \%$ significance, i.e. $90 \%$ confidence interval [CI]) and (ii) comparison of maintenance: PFS with maintenance treatment (superiority comparison target hazard ratio $[\mathrm{HR}]=0.67$, two-sided $20 \%$ significance, i.e. $80 \% \mathrm{CI}$ )

Secondary endpoints included: neuropathy grade $\geq 3$, or grade $\geq 2$ with pain (induction comparison only); complete responses (CR) and overall response rate (ORR); time to maximum response; duration of response; minimal residual disease (MRD); OS; time to next treatment (TTNT); safety/toxicity; and treatment compliance.

Responses were defined according to International Myeloma Working Group (IMWG) guidelines. ${ }^{16}$ Safety and toxicity data were graded using National Cancer Institute (NCI) Common Terminology Criteria for Adverse Events (CTCAE) v4.0. Flow cytometry to detect MRD was performed as previously report$\mathrm{ed}^{17}$ with a limit of detection of $10^{-5}$, assessing 500,000 cells using six-color antibody combinations. Samples classified as "suspicious" (the sample was likely to be positive but there was insufficient evidence) were designated as not MRD negative. ${ }^{18}$ Central assessment of genetic risk was performed as previously described, ${ }^{19}$ supplemented, where appropriate, by local reports that were centrally reviewed. Genetic high risk was defined as at least one of del(17p), gain(1q), or any adverse IgH translocation: $\mathrm{t}(4 ; 14), \mathrm{t}(14 ; 16)$, or $\mathrm{t}(14 ; 20)$.

Total sample sizes of 300 patients for the induction comparison (200 KCd:100 VCd) and 140 for the maintenance comparison were required. ${ }^{15}$ All analyses were pre-planned, unless specified. The analysis population was defined as all participants who received at least one full cycle of allocated chemotherapy. The primary induction endpoint was analyzed using logistic regression, adjusting for minimization factors, with $\mathrm{KCd}$ declared non-inferior to VCd if the $90 \%$ confidence interval for the odds ratio was above 0.8 . PFS, time to maximum response, duration of response, OS and TTNT were analyzed using Kaplan-Meier curves, a log-rank test and Cox proportional hazards models, adjusting for minimization factors. Carfilzomib maintenance was declared superior to no maintenance if the PFS hazard ratio was $<0.67$ and significant at a two-sided $20 \%$ level. TTNT was also analyzed using cumulative incidence 
function curves and Fine and Gray modeling, ${ }^{20}$ considering deaths as competing risks (exploratory).

For the induction comparison of PFS and OS, inverse probability of censoring weighted methods ${ }^{21}$ were used to provide an unbiased comparison of $\mathrm{KCd}$ without maintenance, compared to VCd (Online Supplementary Table S1). The proportions of participants experiencing neuropathy grade $\geq 3$ or grade $\geq 2$ with pain, $C R, O R R$ and $M R D$ status were analyzed using logistic regression, adjusting for minimization factors. Safety, toxicity and treatment compliance were summarized descriptively. As per trial design, $90 \%$ confidence intervals were calculated for induction endpoints and $80 \%$ confidence intervals for PFS. ${ }^{15}$ For all other maintenance endpoints, 95\% confidence intervals were calculated. Induction comparisons were analyzed to evaluate non-inferiority; therefore, $P$-values for these results are only provided to aid interpretation where superiority is observed.

\section{Results}

Induction comparison ( $\mathrm{KCd}$ vs. VCd)

Between February 2013 and September 2016, 300 participants from 35 UK centers were randomized between $\mathrm{KCd}(\mathrm{n}=201)$ and VCd (n=99) (Figure 2). The final analysis took place in two parts with short-term endpoints up to 24 weeks after the initial randomization analyzed as of May 3, 2017 (median follow-up 11.6 months), and remaining endpoints analyzed as of January 3, 2018 (median follow-up from initial randomization 13.9 months: KCd 15 months, VCd 12.6 months). Overall, 196 $\mathrm{KCd}$ and $96 \mathrm{VCd}$ participants were eligible for the safety and efficacy analyses. The baseline characteristics of the patients in the two treatment arms (Table 1) were similar, with around $20 \%$ having been exposed to bortezomib and/or lenalidomide.

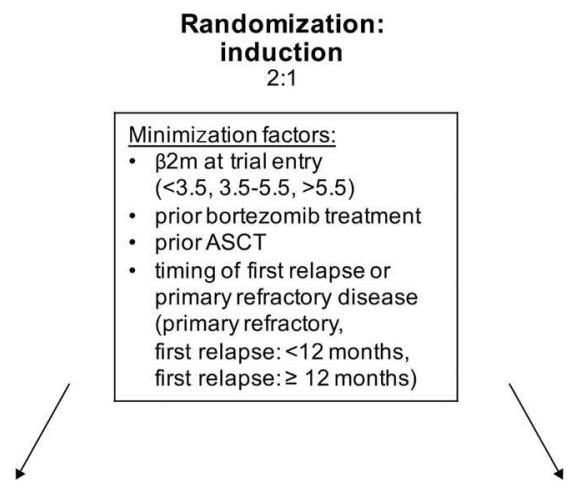

KCd

$6 \times 28$ day cycles $=24$ weeks $^{*}$

\begin{tabular}{|l|l|l|}
\hline Carfilzomib (K) & IV & $\begin{array}{l}20 \mathrm{mg} / \mathrm{m}^{2} \\
\text { (cycle } 1 \text { days 1 \& 2 only) } \\
36 \mathrm{mg} / \mathrm{m}^{2} \\
\text { (days 1, 2, 8, 9, 15, 16) }\end{array}$ \\
\hline $\begin{array}{l}\text { Cyclophosphamide } \\
\text { (C) }\end{array}$ & Oral & $\begin{array}{l}500 \mathrm{mg} \\
\text { (days 1, 8, 15) }\end{array}$ \\
\hline Dexamethasone (d) & Oral & $\begin{array}{l}40 \mathrm{mg} \\
\text { (days 1, 8, 15, 22) }\end{array}$ \\
\hline
\end{tabular}

*Until disease progression, intolerance or a maximum of 6 cycles

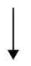

Randomization:

maintenance

$1: 1$

In the absence of disease progression

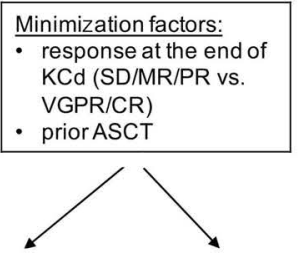

Carfilzomib maintenance

No maintenance therapy

$8 \times 28$ day cycles $=18$ month

\section{\begin{tabular}{l|l|l} 
Carfilzomib & IV & $36 \mathrm{mg} / \mathrm{m}^{2}$
\end{tabular}}

(K)

(days $1,2,15,16$ for 6 months;

days 1,2 , for a further 12 months)

*Until disease progression, intolerance or a maximum of 18 months

VCd

$8 \times 21$ day cycles $=24$ weeks $*$

\begin{tabular}{|l|l|l|}
\hline Bortezomib (V) & SC & $\begin{array}{l}1.3 \mathrm{mg} / \mathrm{m}^{2} \\
\text { (days 1, 4, 8, 11) }\end{array}$ \\
\hline $\begin{array}{l}\text { Cyclophosphamide } \\
\text { (C) }\end{array}$ & Oral & $\begin{array}{l}500 \mathrm{mg} \\
\text { (days 1, 8, 15) }\end{array}$ \\
\hline Dexamethasone (d) & Oral & $\begin{array}{l}40 \mathrm{mg} \\
\text { (days 1, 8, 15, 22) }\end{array}$ \\
\hline
\end{tabular}

*Until disease progression, intolerance or a maximum of 8 cycles

Figure 1. Study randomization and treatment schema. The MUKfive study had two randomizations, one at study registration and another for participants in the carfilzomib, cyclophosphamide, dexamethasone (KCd) arm who achieved at least stable disease after 24 weeks of KCd. Minimization factors are indicated. ASCT: autologous stem cell transplantation; B2m: $\beta$ macroglobulin; IV: intravenous; SC: subcutaneous; SD: stable disease; MR: minimal response; PR: partial response; VGPR: very good partial response; CR: complete response. 


\section{Response to treatment}

Response at 24 weeks was available for 285 participants. More participants receiving $\mathrm{KCd}$ achieved $\geq \mathrm{VGPR}$ compared to those receiving VCd (40.2\% vs. $31.9 \%$, for a difference of $8.3 \%$ [90\% CI: $-1.6,18.2]$, non-inferior). In logistic regression modeling of $\geq V G P R$ at 24 weeks, the odds ratio was 1.48 (90\% CI: 0.95, 2.31), demonstrating non-inferiority (Table 2A). Analysis of patient- and disease-related factors showed broadly similar effects across subgroups (Figure 3A). The proportion of participants
Enrollment, Randomization 1

Follow-Up 1

Analysis

Randomization 2

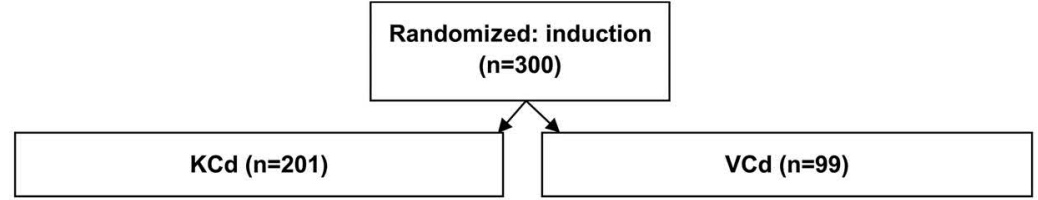

- Lost to follow-up $(n=0)$

- Received no treatment $(n=5)$ - Clinician decision $(n=2)$ - Withdrawal of consent $(n=1)$ o Other $(n=2)$

- Discontinued intervention $(n=196)$ -Maximum \# cycles $(n=160)$ - Toxicity $(n=14)$

Disease progression ( $n=13)$ - Suboptimal response $(n=4)$ o Death $(n=5)$

- Clinician decision lother $(n=3)$ - Withdrawal of consent ( $n=4)$ $\downarrow$

Analyzed ( $n=196)$

- Safety and efficacy analyses $(n=196)$

- Lost to follow-up $(n=0)$

- Received no treatment $(n=3)$ o Withdrawal of consent $(n=3)$

- Discontinued intervention $(n=96)$

- Maximum \# cycles $(n=54)$

- Toxicity $(n=19)$

-Disease progression $(n=6)$

- Suboptimal response $(n=11)$

o Death $(n=1)$

- Clinician decision lother $(n=7)$

- Withdrawal of consent $(n=8)$

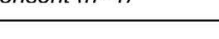

Analyzed $(n=96)$

- Safety and efficacy analyses ( $n=96)$

Not randomized $(n=55)$

○Death $(n=7)$

- Disease progression $(n=20)$

- Received $<5$ cycles $K C d(n=8)$

Withdrew from further treatment

$(n=13)$

- Maximum response not achieved

$(n=1)$

- Eligibility criteria not met $(n=3)$

- Received transplant $(n=3)$

Randomized: maintenance $(n=141)$

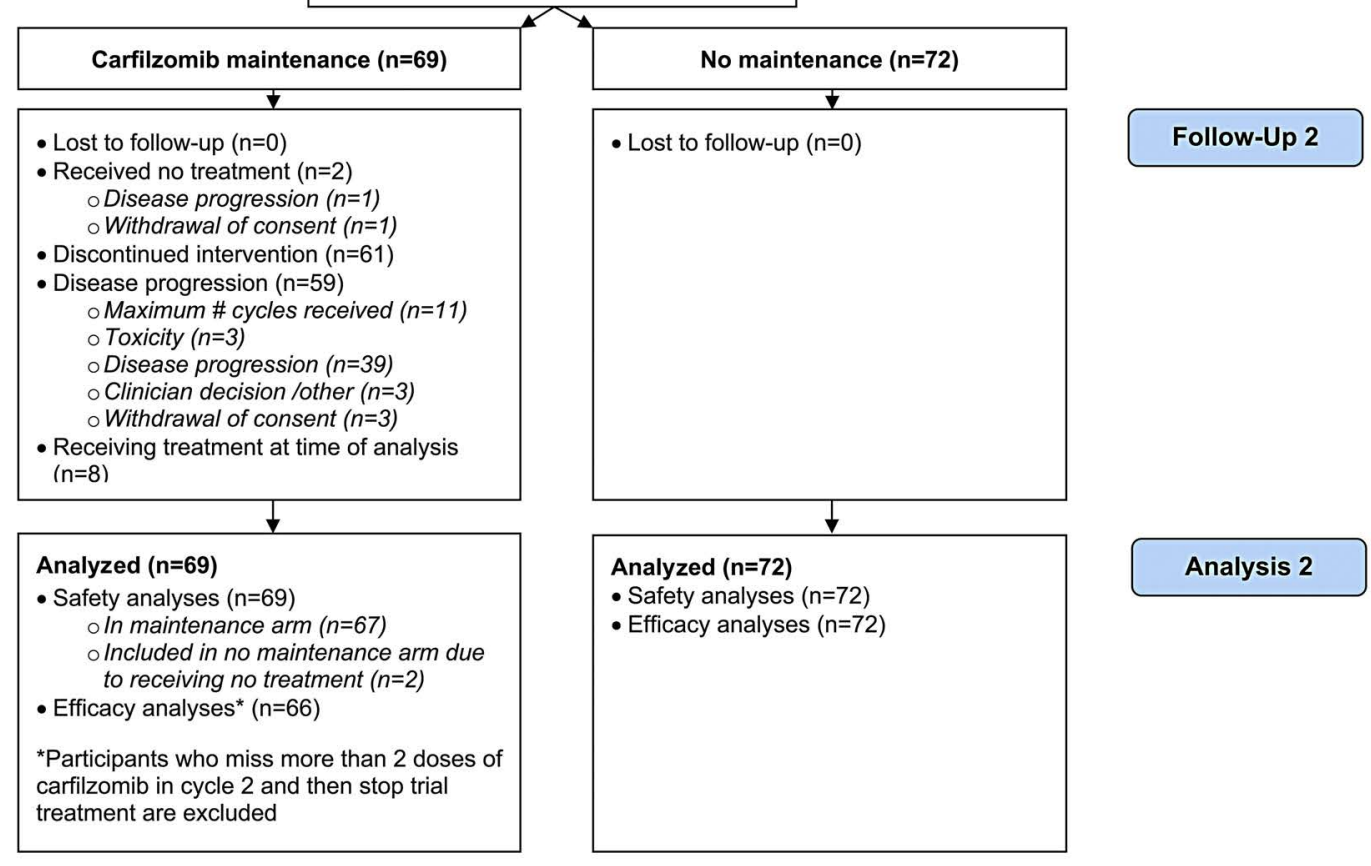

Figure 2. CONSORT (Consolidated Standards of Reporting Trials) diagram. The patients' flow through the study, according to randomization points and treatment arms. KCd: carfilzomib, cyclophosphamide, dexamethasone; VCd: bortezomib, cyclophosphamide, dexamethasone. 
achieving an objective response (i.e., $\geq$ partial response) at 24 weeks was higher for $\mathrm{KCd}$ than for $\mathrm{VCd}(84.0 \%$ vs. $68.1 \%$, respectively), with a difference of $15.9 \%(90 \% \mathrm{CI}$ : $6.8,25.0)$. In logistic regression modeling of overall response at 24 weeks, the odds ratio was 2.72 (90\% CI: $1.62,4.55 ; P=0.0014)$. Subgroup analysis (Figure $3 \mathrm{~B}$ ) indicated broadly similar treatment effects across all subgroups, except that patients not treated with ASCT and those relapsing within 12 months had particular benefit from carfilzomib (interaction $P<0.05$ for both).

Similar results were observed for ORR and VGPR within 12 months (Online Supplementary Table S2). Participants in the $\mathrm{KCd}$ arm had significantly longer time to maximum response (median 2.9 vs. 2.2 months for $\mathrm{VCd}$ : $\mathrm{HR}=0.74$, 90\% CI: $0.60,0.92 ; P=0.0220)$. The median duration of response was 11.1 months for $\mathrm{KCd} v s .10 .1$ months for $\operatorname{VCd}(\mathrm{HR}=0.87$ and $90 \% \mathrm{CI}: 0.64,1.17 ; P=0.441)$.

MRD was assessed at 24 weeks in 157 samples that were received and evaluable, 121 for the $\mathrm{KCd}$ arm, and 46 for the VCd arm. For KCd, 22 (18.2\%) participants were MRD negative, compared to six $(13.0 \%)$ for $\mathrm{VCd}$ (OR=1.48, 90\% CI: 0.64, 3.40) (Online Supplementary Table S3).

\section{Progression-free survival, overall survival and time} to next treatment

All participants in the analysis population were included in the inverse probability of censoring weighted PFS analysis ( $\mathrm{n}=169$ events in participants not weighted 0 ) (Online Supplementary Table S1). The median PFS in the

A

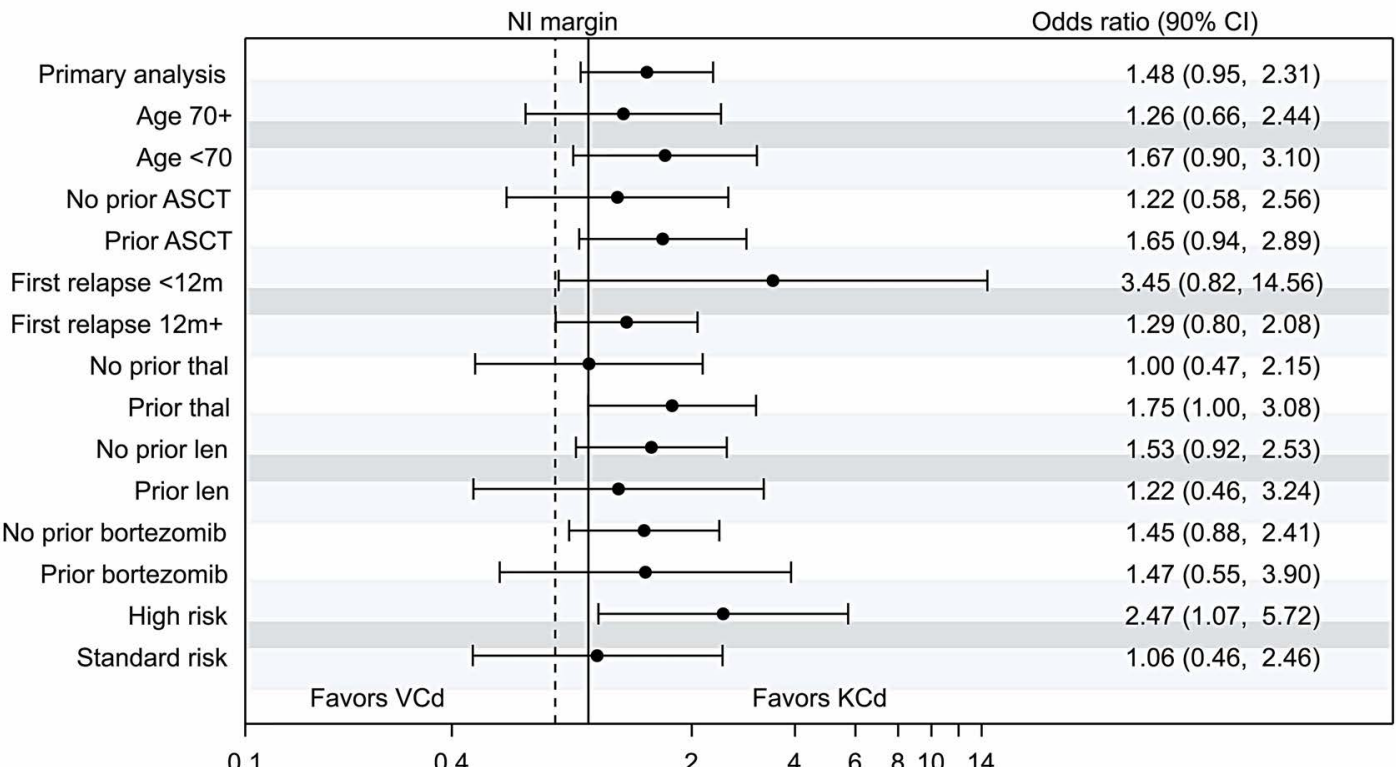

B

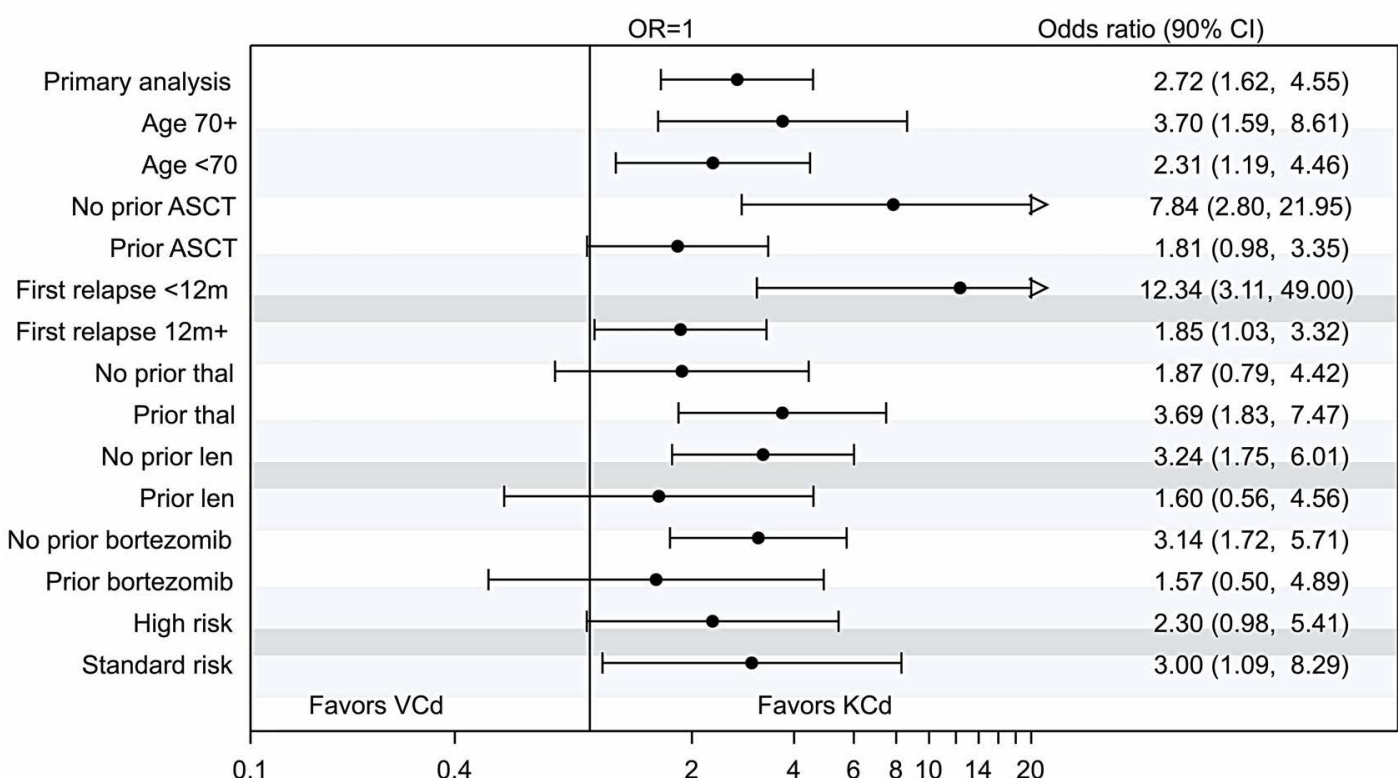

Figure 3. Forest plots of responses at 24 weeks after the initial randomization. The Forest plots show results of an analysis of (A) very good partial response and (B) overall response rate in pre-specified subgroups of the intention-to-treat population, at 24 weeks after the initial randomization. The odds ratio (OR) is provided from logistic regression modeling. NI: non-inferiority; Cl: confidence interval; ASCT: autologous stem cell transplantation; $12 \mathrm{~m}$ : 12 months; thal: thalidomide; len: lenalidomide; KCd: carfilzomib, cyclophosphamide, dexamethasone; VCd: bortezomib, cyclophosphamide, dexamethasone. 
KCd arm was 11.7 months versus 10.2 months in the VCd $\operatorname{arm}(\mathrm{HR}=0.95,80 \% \mathrm{CI}: 0.77,1.18)$ (Figure 4A). Factors prognostic for longer PFS were no previous ASCT and lower $\beta_{2}$-microglobulin level (Online Supplementary Table S4). PFS was not significantly different between treatment arms; however, subgroup analysis suggests that patients relapsing earlier and/or who had not received ASCT may benefit from $\mathrm{KCd}$ therapy (Figure 4B). For analysis of TTNT, 66 participants in the $\mathrm{KCd}$ arm were censored at maintenance randomization. The median TTNT in the KCd arm was 19.1 months, compared with 17.7 months in the VCd arm.

All participants in the analysis population were included in the OS analysis $(n=59$ events in participants not weighted 0 ). The median (inverse probability of censoring weighted) OS with KCd was 30.9 months versus 28.1 months with VCd ( $\mathrm{HR}=1.10,90 \% \mathrm{CI}$ : 0.68, 1.80) (Online Supplementary Figure S1).

\section{Safety and tolerability of induction treatment}

The number of participants who received the planned 24 weeks of treatment was 164 of $201(81.6 \%)$ in the $\mathrm{KCd}$ arm, compared to 53 of $99(53.5 \%)$ in the $\mathrm{VCd}$ arm. Among the patients receiving $\mathrm{KCd}, 14$ (7.0\%) stopped treatment because of toxicity, compared to $19(19.2 \%)$ of those receiving VCd (Online Supplementary Table S5). In the $\mathrm{KCd}$ arm, 11 (5.4\%) subjects stopped treatment because of the patients' withdrawal or clinicians' decision, compared to $20(20 \%)$ in the VCd arm (Online Supplementary Table S5). Dose modifications were reported for $78.6 \%$ of $\mathrm{KCd}$ participants, compared to $85.4 \%$ for VCd (Online Supplementary Table S6). Neuropathy (grade $\geq 3$, or $\geq 2$ with pain) was more common with VCd $(19.8 \%)$ than with $\mathrm{KCd}(1.5 \%)$ for a proportional difference of $-18.3(90 \% \mathrm{CI}$ -25.1, -11.4; $P<0.0001$ ) (Online Supplementary Table S7A, B).

There were 142 serious adverse events in 88 (44.9\%) participants in the $\mathrm{KCd}$ arm, compared with 74 events in

A

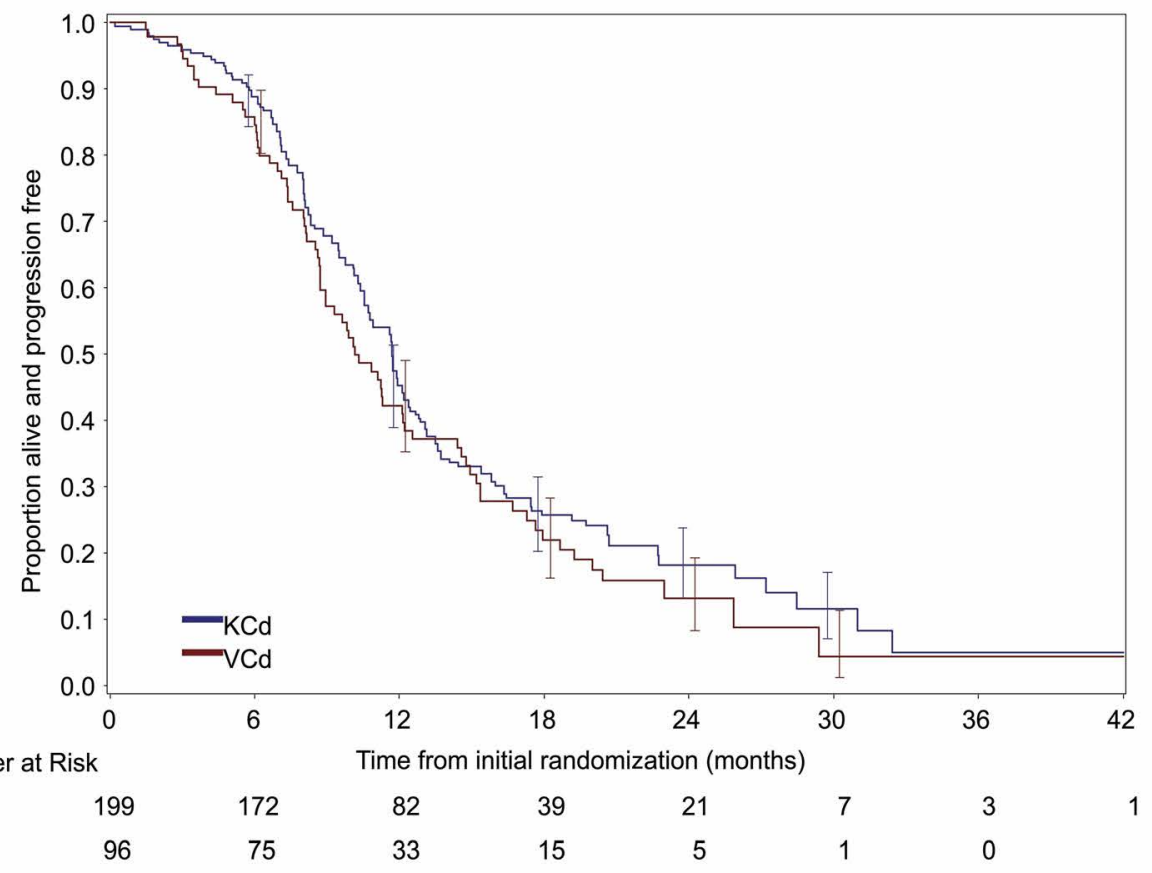

B

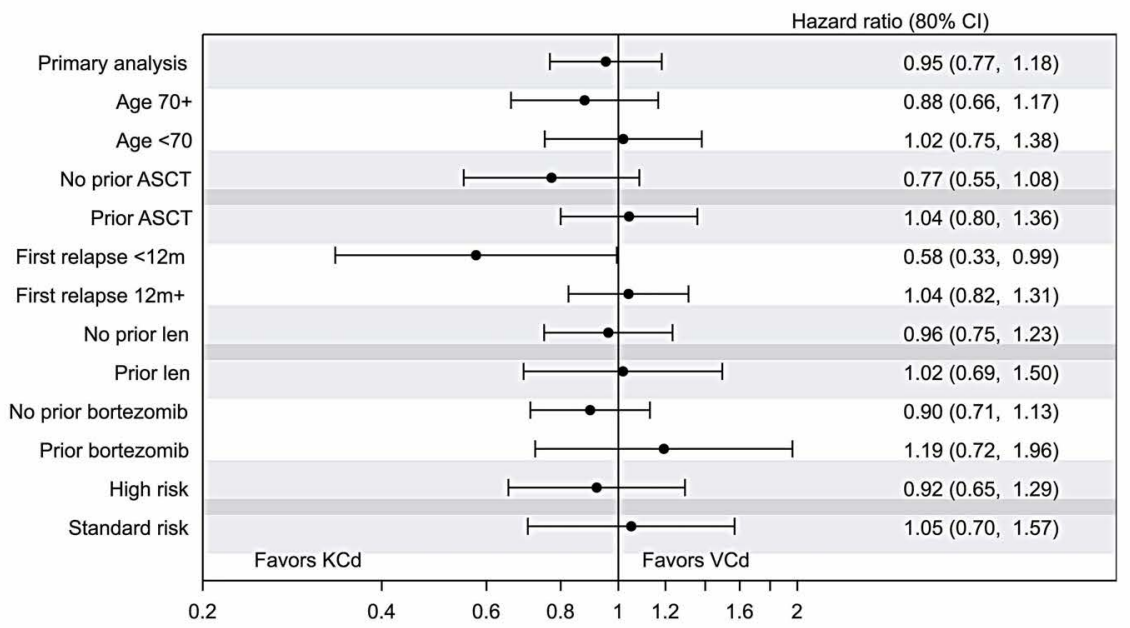

Figure 4. Progression-free survival according to induction randomization. (A) The inverse probability of censoring weighted methodology was used to provide estimates of the progression-free survival (PFS), which is shown with $80 \%$ confidence intervals. (B) Results are shown of an analysis of PFS in pre-specified subgroups of the intention-to-treat population. Forest plot hazard ratios were provided from Cox proportional hazards modeling, using the inverse probability of censoring weighted methodology. KCd: carfilzomib, cyclophosphamide, dexamethasone; VCd: bortezomib, cyclophosphamide, dexamethasone; ASCT: autologous stem cell transplantation; $12 \mathrm{~m}$ : 12 months; len: Ienalidomide; $\mathrm{Cl}$ : confidence interval. 
$45(46.9 \%)$ participants in the VCd arm (Online Supplementary Table S8A). Serious adverse events reported more frequently with $\mathrm{KCd}$ included cardiac, respiratory/thoracic, vascular and renal events while neurological and blood/lymphatic events were more frequent with VCd. The grade $\geq 3$ adverse reactions that occurred are summarized in Online Supplementary Table $S 8 B, C$. More participants in the VCd arm experienced thrombocytopenia and neutropenia, but more in the $\mathrm{KCd}$ arm experienced anemia, hyponatremia and hypophosphatemia. Cardiac adverse reactions were similar in both arms, although grade 3 events were only reported in the $\mathrm{KCd}$ arm ( $\mathrm{n}=6,3.6 \%$ ). Hypertension grade $\geq 3$ was only reported among patients receiving $\mathrm{KCd}$ (3.6\%). Infections accounted for around half of the serious adverse events, with rates being similar in the two arms. There were six deaths during the induction phase, of which five were in the $\mathrm{KCd}$ arm. Safety and treatment tolerability were not influenced by age ( $\geq 70$ years) or renal impairment (glomerular filtratrion rate $\leq 60 \mathrm{~mL} / \mathrm{min}$ ) (Online Supplementary Table S9).

\section{Maintenance comparison}

A total of 141 participants were randomized between maintenance $(n=69)$ and no maintenance $(n=72)$ between August 2013 and March 2017. The data lock took place on January 3, 2018 (median follow-up from maintenance randomization 10.5 months: maintenance 12.7 , observation 7.3). All participants were eligible for the safety analysis: two participants randomized to maintenance did not actually receive the maintenance therapy and were included in the no maintenance arm for the safety analysis (Figure 2). Sixty-six patients given maintenance and 72 not given maintenance were eligible for the efficacy analysis. The patients in the two arms were balanced for baseline characteristics at study entry, and disease response at the time of maintenance randomization (Table 3 ). The median number of cycles received in the maintenance arm was

A

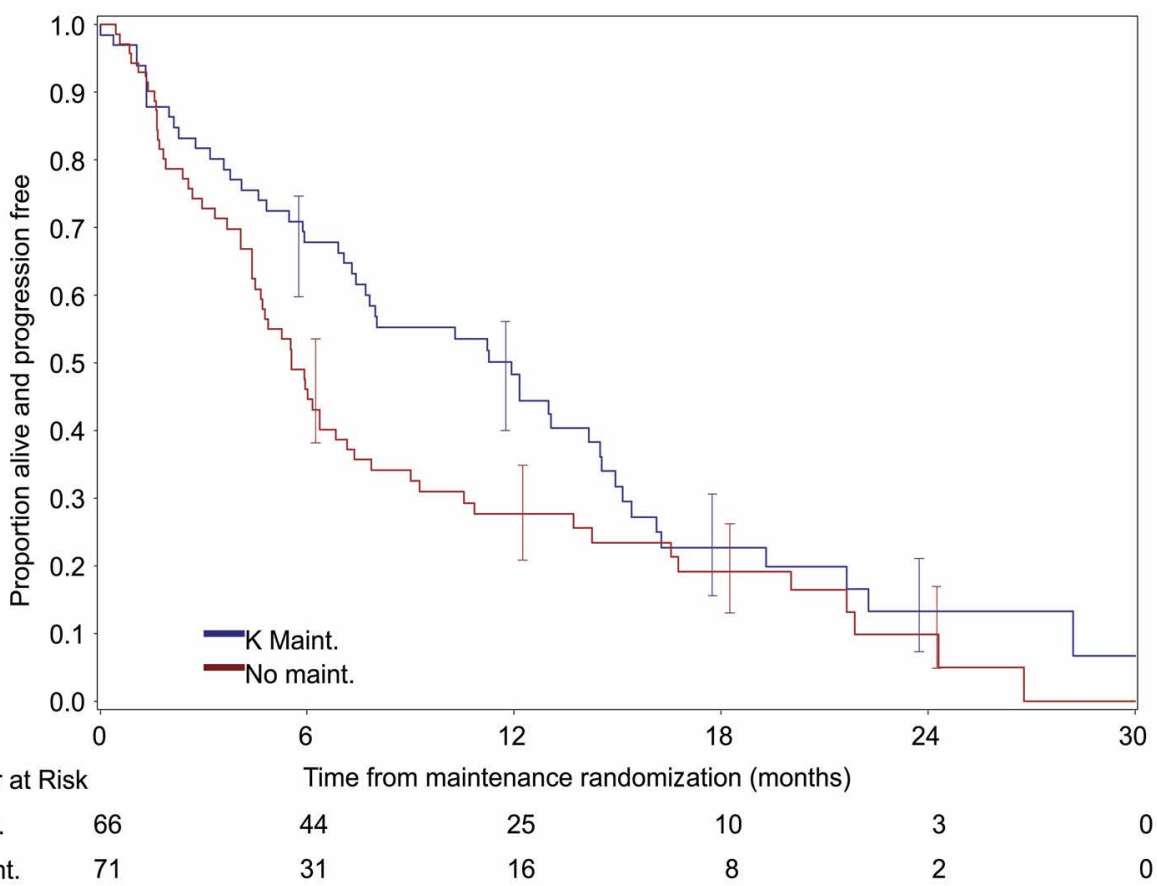

B

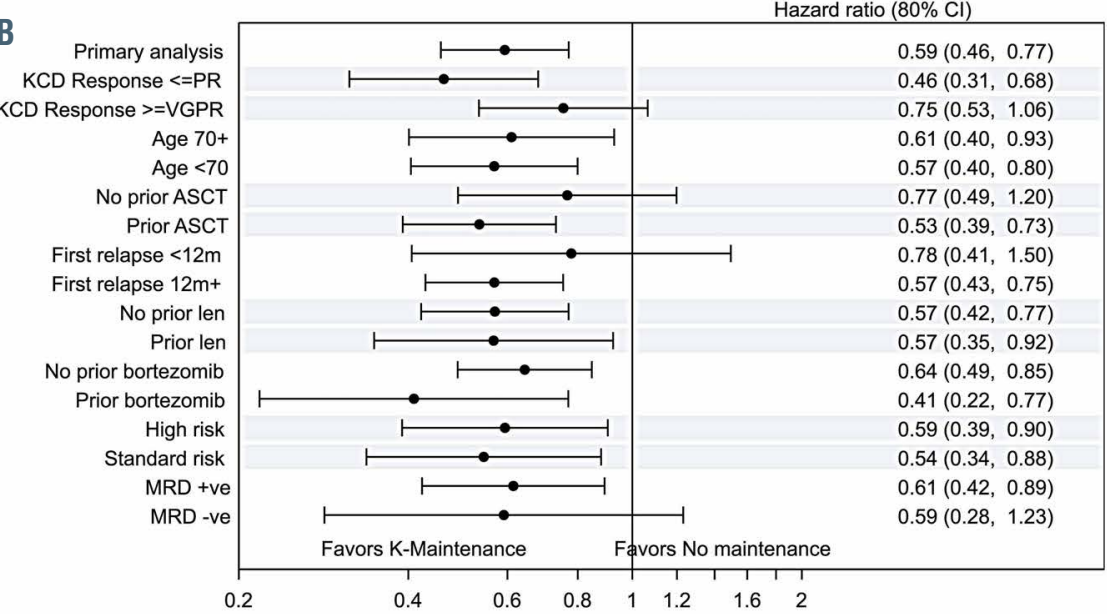

Figure 5. Progression-free survival according to maintenance randomization. (A) Kaplan-Meier estimate of progression-free survival (PFS), shown with $80 \%$ confidence intervals, and numbers at risk indicated. (B) Forest plot of PFS in pre-specified subgroups, with hazard ratios and $80 \%$ confidence intervals. $\mathrm{K}$ Maint: maintenance with carfilzomib; No maint: no maintenance therapy; Cl: confidence interval; KCd: carfilzomib, cyclophosphamide, dexamethasone; VCd: bortezomib, cyclophosphamide, dex amethasone; ASCT, autologous stem cell transplantation; 12m: 12 months; len: lenalidomide;.KCD: carfilzomib, cyclophosphamide, dexamethasone; PR: partial response; VGPR: very good partial response; ASCT: autologous stem cell transplantation; $12 \mathrm{~m}$ : 12 months; len: lenalidomide; MRD: minimal residual disease. 
eight (range, 0-19). At the time of analysis, eight patients were still on treatment, 61 had stopped, of whom 11 patients received the maximum of 18 cycles, and 23 $(37.7 \%)$ had received fewer than six cycles.

\section{Progression-free survival}

All participants in the analysis population were included in the PFS analysis, with the exception of one patient who progressed prior to maintenance treatment. A total of 107 events were observed (104 disease progression, 3 deaths). Patients in the carfilzomib maintenance arm had significantly longer PFS (median 11.9 months, 80\% CI: 8.0, 13.1), than those who did not receive maintenance therapy (median 5.6 months, $80 \%$ CI: 4.8, 6.4; HR=0.59, $80 \%$ CI: 0.46, 0.77; $P=0.0086$ ) (Figure 5A). Disease response at the time of randomization ( $\geq \mathrm{VGPR})$ was also significantly associated with longer PFS ( $\mathrm{HR}=0.42,80 \%$ CI: 0.32, 0.55; $P<0.0001)$ (Online Supplementary Table S10). The benefit of maintenance was seen across all subgroups

Table 1. Minimization factors for induction randomization, and baseline characteristics.

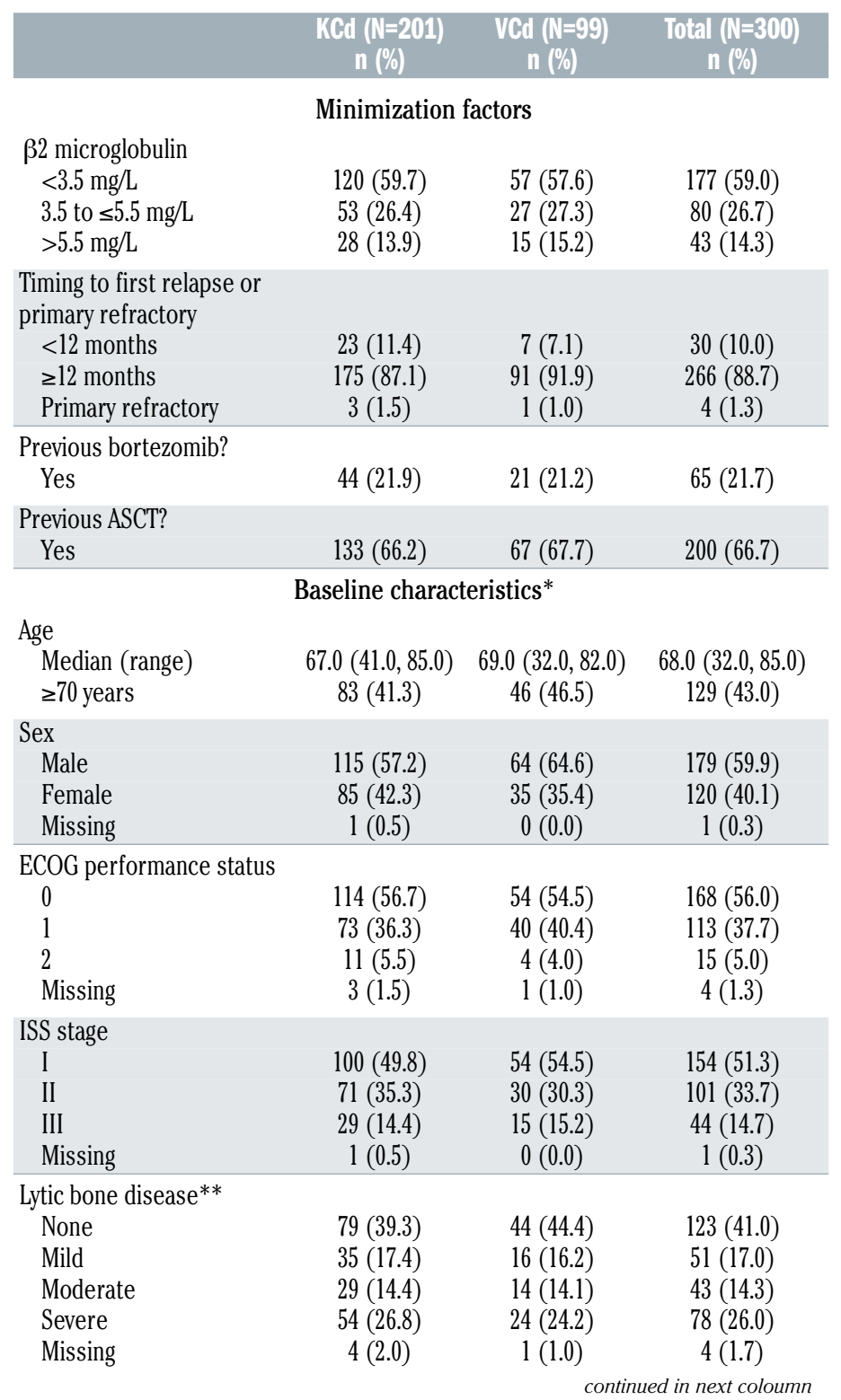

(Figure 5B) and also in patients over 70 years old, who accounted for more than $40 \%$ of the participants. Treatment effects were larger for patients whose response to initial therapy was partial response or less or those who had had a prior ASCT. It is also worth noting that treatment effects were similar between patients in standard- and high-risk genetic subgroups, and did not differ according to MRD status at the end of initial treatment. No significant interactions were observed between subgroups and maintenance treatment.

\section{Overall survival, time to next treatment and response depth: maintenance randomization}

At the time of analysis, 26/138 participants had died. The median OS from the time of maintenance randomization was 25.7 months (95\% CI: 20.8, upper limit not estimated) for maintenance and 24.1 months (95\% CI: 21.5, upper limit not estimated) for observation ( $\mathrm{HR}=0.86,95 \%$ CI: $0.39,1.87 ; P=0.6965)$. The median time from maintenance randomization to next treatment in the maintenance arm was 21.4 months (95\% CI: 20.3, upper limit not estimated) whereas it was 12.9 months (95\% CI: 8.3-27.5) in the control group (Fine and Gray HR=0.59, 95\% CI: 0.34-1.02; $P=0.0566)$.

continued from previous coloum

\begin{tabular}{lccc} 
Heavy chain paraprotein type & & & \\
IgG & $128(63.7)$ & $63(63.6)$ & $191(63.7)$ \\
IgA & $47(23.4)$ & $20(20.2)$ & $67(22.3)$ \\
IgM & $0(0.0)$ & $1(1.0)$ & $1(0.3)$ \\
IgD & $0(0.0)$ & $1(1.0)$ & $1(0.3)$ \\
Light chain only & $25(12.4)$ & $14(14.1)$ & $39(13.0)$ \\
Missing & $1(0.5)$ & $0(0.0)$ & $1(0.3)$ \\
\hline Light chain type & & & \\
Kappa & $136(67.7)$ & $64(64.6)$ & $200(66.7)$ \\
Lambda & $64(31.8)$ & $35(35.4)$ & $99(33.0)$ \\
Missing & $1(0.5)$ & $0(0.0)$ & $1(0.3)$ \\
Previous lenalidomide? & & & \\
Yes & $45(22.5)$ & $23(23.2)$ & $68(22.7)$ \\
\hline Other previous treatments & & & \\
regimens & & & \\
TD/CTD & $122(60.7)$ & $68(68.7)$ & $190(63.3)$ \\
VDNCD/PAD & $33(16.4)$ & $18(18.2)$ & $51(17.0)$ \\
MP/MPTNMP & $7(3.5)$ & $1(1.0)$ & $8(2.7)$ \\
Genetic risk*** (n=187) & & & \\
High risk & $69(55.6)$ & $33(52.4)$ & $102(54.5)$ \\
Standard risk & $55(44.4)$ & $30(47.6)$ & $85(45.5)$ \\
Total with confirmed & & & \\
risk status & 124 & 63 & 187 \\
\hline High-risk lesions (n=102) & & & \\
Del(17p) only & $11(15.9)$ & $6(18.2)$ & $17(16.7)$ \\
Gain(lq) only & $43(62.3)$ & $19(57.6)$ & $62(60.8)$ \\
Any adverse IGH translocation & & & \\
only & $4(5.8)$ & $4(12.1)$ & $8(7.9)$ \\
Gain(1q) and del(17p) & $4(5.8)$ & $1(3.0)$ & $5(4.9)$ \\
Gain(lq) and any adverse IGH & & & \\
translocation & $6(8.7)$ & $2(6.0)$ & $8(7.9)$ \\
Gain(lq), del(17p) and t(4;14) $)$ & $1(1.4)$ & $1(3.0)$ & $2(2.0)$ \\
\hline
\end{tabular}

* One participant in the $\mathrm{KCd}$ arm was found to be ineligible after randomization, therefore no subsequent data were collected, including baseline characteristics. * ${ }^{*}$ Mild $=$ one fracture (any site including vertebrae) or lytic lesion. Moderate $=$ two or three fractures (any site including vertebrae) or lytic lesions. Severe $=$ three or more fractures (any site including vertebrae) or lytic lesions. ${ }^{* *}$ Genetic high risk was defined as at least one of del(17p), gain(1q), or any adverse IgH translocation: $\mathrm{t}(4 ; 14), \mathrm{t}(14 ; 16)$, or $\mathrm{t}(14 ; 20)$. KCd: carfilzomib, cyclophosphamide, dexamethasone; VCd: bortezomib, cyclophosphamide, dexamethasone; ASCT: autologous stem cell transplantation; ECOG: Eastern Cooperative Oncology Group; ISS: International Staging System. 
Maintenance improved disease response in 13 participants: one patient with stable disease had a minimal response, eight with partial responses developed $\geq V G P R$, and four with VGPR achieved complete responses. In the control arm, eight participants' response deepened: seven improved from having partial responses to VGPR and one with a VGPR achieved a complete response. Thus, in the maintenance arm, $10.6 \%$ patients achieved a complete response and $50.0 \%$ a VGPR as their maximum response overall, compared to $4.2 \%$ and $55.6 \%$ respectively, in the control arm. Maintenance also increased the rate of MRD negativity (Online Supplementary Figure S2). Maintenance was significantly associated with a higher MRD negative rate at 6 months $(24.4 \%$ vs. $3.3 \%$; OR=9.66, 95\% CI: 1.17 , 80.02; $P=0.0071)$. The difference was not statistically significant at 12 months.

\section{Safety and tolerability of carfilzomib maintenance}

Of the 50 patients who stopped carfilzomib maintenance therapy early, $40(80.0 \%)$ did so because of disease progression, three stopped due to toxicity (after 5, 6, and 17 cycles), and seven due to the patients' or clinicians' choice. Dose modifications were reported for 55 of 67 participants who received treatment (82.1\%), with $11.3 \%$ of cycles being delayed. The median dose received was $36 \mathrm{mg} / \mathrm{m}^{2}$ (target dose). Thirty-four serious adverse events were reported in 24 participants receiving maintenance: 19 serious adverse events, 15 serious adverse reactions (infection or infestation $[n=11]$; gastrointestinal, cardiac, renal and secondary primary malignancy $[\mathrm{n}=1$ each), no suspected unexpected adverse reaction. In the group not receiving maintenance, six serious adverse events were reported in six participants. Most events $(90 \%)$ resolved ( 1 serious adverse event was present and unchanged, 1 resulted in death, 2 were ongoing at the time of death). Adverse reactions are summarized in Online Supplementary Table S11. Among adverse reactions of interest, there was one cardiac adverse reaction (grade 2, chest pain), six cases of acute kidney injury (1 grade 3), four of hypertension (2 grade 3), 25 upper respiratory tract/bronchial infections (1 grade 3$)$, and six lung infections (3 grade 3$)$. Toxicity did not appear to increase over time.

\section{Landmark analysis of VCd versus $\mathrm{KCd}-\mathrm{K}$ versus $\mathrm{KCd}$}

A post-hoc landmark analysis was performed at 6 months after first randomization to assess PFS for those patients who had not progressed at this time, comparing VCd with KCd plus maintenance (with carfilzomib), and $\mathrm{KCd}$ with no maintenance. The median PFS for patients receiving VCd was 6.6 months (95\% CI: 4.6, 9.7), similar to that for patients receiving $\mathrm{KCd}$ without maintenance (6.2 months, 95\% CI: 5.2, 8.0) (Online Supplementary Figure $S 3)$. For patients receiving maintenance therapy the median PFS was 12.6 months (95\% CI: 8.4, 15.0), reflecting that observed in the primary analysis. The PFS from initial randomization for patients receiving $\mathrm{KCd}$ followed by carfilzomib maintenance was also evaluated: among these patients, the median PFS was 18.1 months (95\% CI: 14.0, 20.5).

\section{Influence of genetic risk}

Genetic risk status at trial entry was available for 187 (62.3\%) participants; $55.6 \%$ and $52.4 \%$ of participants in the KCd and VCd arms, respectively, had adverse-risk genetics (Table 1). Among participants with adverse risk, $78.3 \%$ and $63.6 \%$ received the planned six cycles of $\mathrm{KCd}$ and eight cycles of VCd, respectively, compared to $92.7 \%$ and $43.3 \%$ of the standard-risk participants (Online Supplementary Table S12). The reason for this difference is unclear, but a greater proportion of standard-risk participants in the VCd arm discontinued therapy.

With respect to the initial treatment, more participants with adverse risk achieved $\geq V G P R$ (over 24 weeks) on $\mathrm{KCd}$ compared with VCd (38.2\% vs. $21.9 \%$, OR=2.47, $90 \%$ CI: 1.07, 5.72), while standard-risk participants had a similar $\geq$ VGPR rate in the two arms (KCd $34.5 \%$, VCd $33.3 \%, P($ interaction $)=0.2425)$ (Online Supplementary Figure

Table 2A. Response to treatment at 24 weeks after the initial randomization.

\begin{tabular}{|c|c|c|c|c|}
\hline \multirow[t]{2}{*}{ Outcome } & \multirow[b]{2}{*}{ KCd } & \multicolumn{3}{|c|}{ KCd vs. VCd comparison } \\
\hline & & VCd & $\begin{array}{c}\text { Difiference (\%), } \\
90 \% \mathrm{Cl}\end{array}$ & $\begin{array}{l}\text { OR, } 90 \% \text { Cl, } \\
\text { P-value }\end{array}$ \\
\hline Participants with available response & 194 & 91 & & \\
\hline 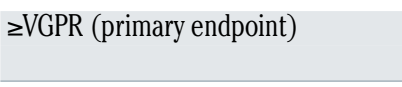 & $40.2 \%$ & $31.9 \%$ & $8.3(-1.6,18.2)$ & $\begin{array}{c}1.48,(0.95,2.31) \\
\text { NI comparison so } P \text {-value not relevant }\end{array}$ \\
\hline Overall response: $\geq P R$ & $84.0 \%$ & $68.1 \%$ & $15.9(6.8,25.0)$ & $2.72,(1.62,4.55), P=0.0014$ \\
\hline Complete response & $1.5 \%$ & $3.3 \%$ & $-1.8(-5.2,1.7)$ & Logistic regression not performed \\
\hline
\end{tabular}

Table 2B. Response to treatment at 24 weeks after initial randomization, by genetic risk.

\begin{tabular}{|c|c|c|c|c|c|c|c|}
\hline \multirow[t]{2}{*}{ Outcome } & \multicolumn{3}{|c|}{ High risk } & \multicolumn{3}{|c|}{ Standard risk } & \multirow{2}{*}{$\begin{array}{c}\text { P-value } \\
\text { for interaction }\end{array}$} \\
\hline & $\mathrm{KCd}$ & VCd & $\begin{array}{l}\text { KCd vs. VCd: } \\
\text { OR (90\% CI) }\end{array}$ & KCd & VCd & $\begin{array}{l}\text { KCd vs. VCd: } \\
\text { OR (90\% CI) }\end{array}$ & \\
\hline Participants with available response & 68 & 32 & & 55 & 27 & & \\
\hline$\geq \mathrm{VGPR}$ & $38.2 \%$ & $21.9 \%$ & $2.47(1.07,5.72)$ & $34.5 \%$ & $33.3 \%$ & $1.06(0.46,2.46)$ & 0.2425 \\
\hline Overall response: $\geq P R$ & $79.4 \%$ & $68.8 \%$ & $2.30(0.98,5.41)$ & $87.3 \%$ & $70.4 \%$ & $3.00(1.09,8.29)$ & 0.7403 \\
\hline Participants with available MRD data & 52 & 17 & & 41 & 15 & & \\
\hline MRD negativity & $17.3 \%$ & $17.6 \%$ & $1.13(0.32,3.95)$ & $12.2 \%$ & $13.3 \%$ & $0.85(0.18,3.99)$ & 0.8153 \\
\hline
\end{tabular}

KCd: carfilzomib, cyclophosphamide, dexamethasone; VCd: bortezomib, cyclophosphamide, dexamethasone; OR: odds ratio; CI: confidence interval;VGPR: very good partial response; PR: partial response; NI: non-inferiority; MRD: minimal residual disease. 
S4A, Table 2B). This difference between risk groups was not seen for the ORR, with there being more responses to $\mathrm{KCd}$ than to $\mathrm{VCd}$ in both adverse- and standard-risk participants, $(79.4 \% v s .68 .8 \%$, and $87.3 \%$ vs. $70.4 \%$ ). Of participants with an adverse IgH translocation, only one of seven $(14.3 \%)$ in the VCd arm achieved a VGPR, compared to seven of $11(63.6 \%)$ in the $\mathrm{KCd}$ arm (Online Supplementary Figure $S 4 B$ ). There was no significant difference in MRD-negative rates (Table 2B) or PFS between the $\mathrm{KCd}$ and $\mathrm{VCd}$ arms in either adverse- or standard-risk patients.

With regard to the maintenance randomization, genetic risk status was available overall for 94 participants $(67 \%)$ (Table 3), with $48.4 \%$ adverse-risk patients in the maintenance arm, and $55.1 \%$ in the observation arm. Of those completing treatment, the standard-risk patients received a median of nine cycles (range, 0-19), compared with five cycles (range, 1-18) for the adverse-risk patients. While 11 of $23(47.8 \%)$ standard-risk patients received $>12$ months

Table 3. Characteristics of patients in the maintenance randomization: at study entry and at randomization.

\begin{tabular}{|c|c|c|c|}
\hline & $\begin{array}{l}\text { Maintenance } \\
\qquad(N=69) \\
n(\%)\end{array}$ & $\begin{array}{l}\text { No maintenance } \\
\begin{array}{c}(N=72) \\
n(\%)\end{array}\end{array}$ & $\begin{array}{c}\text { Total } \\
(N=141) \\
n(\%)\end{array}$ \\
\hline \multicolumn{4}{|c|}{ Baseline characteristics at initial randomization } \\
\hline $\begin{array}{l}\text { Age at trial entry } \\
\text { Median (range) } \\
\geq 70 \text { years }\end{array}$ & $\begin{array}{l}65(35,80) \\
29(42.0 \%)\end{array}$ & $\begin{array}{l}69(48,83) \\
34(47.2 \%)\end{array}$ & $\begin{array}{l}68(35,83) \\
63(44.7 \%)\end{array}$ \\
\hline $\begin{array}{l}\text { Sex } \\
\quad \text { Male } \\
\text { Female }\end{array}$ & $\begin{array}{l}43(62.3 \%) \\
26(37.7 \%)\end{array}$ & $\begin{array}{l}42(58.3 \%) \\
30(41.7 \%)\end{array}$ & $\begin{array}{l}85(60.3 \%) \\
56(39.7 \%)\end{array}$ \\
\hline $\begin{array}{l}\text { Timing of first relapse } \\
\text { Primary refractory } \\
\quad<12 \text { months } \\
\quad \geq 12 \text { months }\end{array}$ & $\begin{array}{c}1(1.4 \%) \\
10(14.5 \%) \\
58(84.1 \%)\end{array}$ & $\begin{array}{c}1(1.4 \%) \\
8(11.1 \%) \\
63(87.5 \%)\end{array}$ & $\begin{array}{c}2(1.4 \%) \\
18(12.8 \%) \\
121(85.8 \%)\end{array}$ \\
\hline $\begin{array}{l}\text { ISS stage } \\
\text { I } \\
\text { II } \\
\text { III }\end{array}$ & $\begin{array}{c}39(56.5 \%) \\
24(34.8 \%) \\
6(8.7 \%)\end{array}$ & $\begin{array}{c}42(58.3 \%) \\
24(33.3 \%) \\
6(8.3 \%)\end{array}$ & $\begin{array}{c}81(57.4 \%) \\
48(34.0 \%) \\
12(8.5 \%)\end{array}$ \\
\hline $\begin{array}{l}\text { Disease isotype } \\
\text { IgG } \\
\text { IgA } \\
\text { Light chain only }\end{array}$ & $\begin{array}{c}45(65.2 \%) \\
18(26.1 \%) \\
6(8.7 \%)\end{array}$ & $\begin{array}{l}50(69.4 \%) \\
14(19.4 \%) \\
8(11.1 \%)\end{array}$ & $\begin{array}{c}95(67.4 \%) \\
32(22.7 \%) \\
14(9.9 \%)\end{array}$ \\
\hline $\begin{array}{l}\text { Light chain type } \\
\text { Kappa } \\
\text { Lambda }\end{array}$ & $\begin{array}{l}46(66.7 \%) \\
23(33.3 \%)\end{array}$ & $\begin{array}{l}51(70.8 \%) \\
21(29.2 \%)\end{array}$ & $\begin{array}{l}97(68.8 \%) \\
44(31.2 \%)\end{array}$ \\
\hline $\begin{array}{l}\text { Received previous bortezomib? } \\
\text { Yes } \\
\text { No }\end{array}$ & $\begin{array}{l}19(27.5 \%) \\
50(72.5 \%)\end{array}$ & $\begin{array}{l}13(18.1 \%) \\
59(81.9 \%)\end{array}$ & $\begin{array}{c}32(22.7 \%) \\
109(77.3 \%)\end{array}$ \\
\hline $\begin{array}{l}\text { Genetic risk at trial entry }(\mathrm{n}=109) \\
\text { High risk } \\
\text { Standard risk } \\
\text { Risk unconfirmed }\end{array}$ & $\begin{array}{c}22(42.3 \%) \\
23(44.2 \%) \\
7(13.5 \%)\end{array}$ & $\begin{array}{c}27(47.4 \%) \\
22(38.6 \%) \\
8(14.0 \%)\end{array}$ & $\begin{array}{l}49(45.0 \%) \\
45(41.3 \%) \\
15(13.8 \%)\end{array}$ \\
\hline $\begin{array}{l}\text { High risk lesions ( } \mathrm{n}=49) \\
\text { Del(17p) only } \\
\text { Gain(1q) only } \\
\text { t( } 4 ; 14) \text { only } \\
\text { Gain(1q) and del(17p) } \\
\text { Gain(lq) and any adverse } \\
\text { IGH translocation } \\
\text { Gain(lq), del(17p) and } \mathrm{t}(4 ; 14)\end{array}$ & $\begin{array}{c}5(22.7 \%) \\
15(68.2 \%) \\
1(4.5 \%) \\
0\end{array}$ & $\begin{array}{c}3(11.1 \%) \\
15(55.6 \%) \\
3(11.1 \%) \\
3(11.1 \%) \\
\\
2(7.4 \%) \\
1(3.7 \%)\end{array}$ & $\begin{array}{c}8(16.3 \%) \\
30(61.2 \%) \\
4(8.2 \%) \\
3(6.1 \%) \\
3(6.1 \%) \\
1(2.0 \%)\end{array}$ \\
\hline
\end{tabular}

of maintenance, only three of 17 (17.6\%) adverse-risk patients did so. In a Cox proportional hazards model for PFS, including genetic risk and MRD, in addition to minimization factors, genetic risk was independently prognostic, alongside treatment and disease response at the time of randomization (genetic risk: $\mathrm{HR}=1.91,80 \% \mathrm{CI}: 1.27$, 2.59; $P=0.0333$ ). No difference in the impact of maintenance on PFS was observed between the risk groups: both benefited from maintenance treatment (Figure 5B, Online Supplementary Figure S5).

\section{Discussion}

Despite three published head-to-head studies of carfilzomib versus bortezomib, ${ }^{4,9-11}$ the relative benefits of one proteasome inhibitor over the other remain unresolved. Here we show that, when used as second-line therapy, carfilzomib is at least as efficacious as bortezomib, in terms of major disease response, when given for a fixed duration in combination with cyclophosphamide and dexamethasone. There were fewer discontinuations among patients treated with $\mathrm{KCd}$ than among those treated with $\mathrm{VCd}$, indicating that KCd was tolerated better than VCd. Carfilzomib was also well tolerated and effective as a maintenance agent, providing a PFS benefit when compared with observation only. No new safety signals were seen, as toxicity profiles reflected previous experience with these proteasome inhibitors, carfilzomib being predominantly associated with anemia and cardiovascular complications while bortezomib was linked to a higher incidence of peripheral neuropathy, thrombocytopenia

continued from previous coloumn

Minimisation factors at maintenance randomisation

Response category at

the end of therapy

VGPR, CR or SCR

$\mathrm{PR}, \mathrm{MR}$ or $\mathrm{SD} / \mathrm{NC}$

$40(58.0 \%)$

$39(54.2 \%)$

$79(56.0 \%)$

Previous ASCT?

$\begin{array}{crrr}\text { Yes } & 46(66.7 \%) & 48(66.7 \%) & 94 \\ & \text { Participant characteristics at maintenance randomization }\end{array}$

$29(42.0 \%)$

$33(45.8 \%)$

$62(44.0 \%)$

ECOG performance status

$\begin{array}{lccc}0 & 43(62.3 \%) & 38(52.8 \%) & 81(57.4 \%) \\ 1 & 22(31.9 \%) & 32(44.4 \%) & 54(38.3 \%) \\ 2 & 1(1.4 \%) & 1(1.4 \%) & 2(1.4 \%) \\ \text { Missing } & 3(4.3 \%) & 1(1.4 \%) & 4(2.8 \%) \\ \text { RD at end of initial treatment } & & & \\ \text { Positive } & 39(56.5 \%) & 42(58.3 \%) & 81(57.4 \%) \\ \text { Negative } & 8(11.6 \%) & 10(13.9 \%) & 18(12.8 \%) \\ \text { Suspicious } & 2(2.9 \%) & 4(5.6 \%) & 6(4.3 \%) \\ \text { No MRD sample } & 13(18.8 \%) & 11(15.3 \%) & 24(17.0 \%) \\ \text { Not evaluable } & 0(0.0 \%) & 1(1.4 \%) & 1(0.7 \%) \\ \text { Inadequate sample } & 7(10.1 \%) & 4(5.6 \%) & 11(7.8 \%) \\ \text { Sponse at time of randomization } & & & \\ \text { CR } & 2(2.9 \%) & 1(1.4 \%) & 3(2.1 \%) \\ \text { VGPR } & 30(43.5 \%) & 29(40.3 \%) & 59(41.8 \%) \\ \text { PR } & 33(47.8 \%) & 39(54.2 \%) & 72(51.1 \%) \\ \text { MR } & 2(2.9 \%) & 2(2.8 \%) & 4(2.8 \%) \\ \text { SD or NC } & 1(1.4 \%) & 0(0.0 \%) & 1(0.7 \%) \\ \text { PD } & 1(1.4 \%) & 1(1.4 \%) & 2(1.4 \%)\end{array}$

ISS: International Staging System; CR: complete response;VGPR: very good partial response; sCR: stringent complete response; PR: partial response; MR: minimal response; SD: stable disease; $\mathrm{NC}$ : no change; PD: progressive disease; ASCT: autologous stem cell transplantation;

ECOG: Eastern Cooperative Oncology Group; MRD: minimal residual disease. 
and neutropenia. Notably, the carfilzomib dose of 36 $\mathrm{mg} / \mathrm{m}^{2}$ in the current study was associated with a relatively low incidence of cardiovascular adverse events (3.6\%) and grade $\geq 3$ hypertension $(3.6 \%)$ when compared with the higher dose of $56 \mathrm{mg} / \mathrm{m}^{2}$ in the ENDEAVOR trial (6.5\% and $9.0 \%$, respectively). ${ }^{4,9}$ Relatively high rates of infectious serious adverse events in both arms indicate that antibiotic prophylaxis may be considered for relapsed patients treated with proteasome inhibitor triplets, at least for the first few cycles.

The broadly equivalent outcomes of carfilzomib and bortezomib when used in fixed duration protocols, as demonstrated in this study, are in line with the results of the CLARION and ENDURANCE studies, indicating that the PFS and OS benefits seen with carfilzomib in ENDEAVOR were related to the greater tolerability of this proteasome inhibitor, facilitating extended therapy. Another contributing factor to the differing results between our study and ENDEAVOR may be the smaller percentage of our cohort who were exposed to bortezomib and who were, thus, less likely to be resistant. Here we extend our observations to show that maintenance with single-agent carfilzomib is effective in prolonging PFS. Taken as a whole, our observations and the results of previous studies suggest that optimal clinical benefit from carfilzomib can be achieved using well-tolerated extended protocols. This notion is further supported by the more recent studies of carfilzomib in combination with the CD38 antibodies, daratumumab and isatuximab, in which outcomes of patients treated at first relapse may indeed be impressive. ${ }^{6,22}$ With increasing use of carfilzomib leading to improved management of the drug's toxicity profile, and considering promising results with weekly dosing, ${ }^{23}$ durable disease control may be achievable with this proteasome inhibitor, especially in the setting of relapsed disease.

Certain features of our cohort of patients may have influenced the comparative tolerability of KCd and VCd. Even with a fixed duration of treatment of 6 months, there were fewer discontinuations with $\mathrm{KCd}$ than with $\mathrm{VCd}$, and a substantial proportion of patients in the VCd arm discontinued due to their own choice or a clinician's decision. While we do not have details of these decisions, it is noteworthy that over half of patients in this study had previously received a thalidomide regimen, hence several may have been especially sensitive to or intolerant of a salvage regimen containing yet another neurotoxic drug, thus accounting for the high rates of discontinuation in the VCd arm.

We demonstrate that carfilzomib maintenance is efficacious regardless of genetic risk, and in renally impaired and older patients. Our findings on the activity and tolerability of carfilzomib maintenance in the RRMM setting add to those from two phase I/II studies in newly diagnosed patients $\geq 65$ years old, or ineligible for transplantation, who received single-agent carfilzomib maintenance (until progression) after nine cycles of $\mathrm{KCd}^{12,13}$ In those studies, responses deepened during the maintenance phase, and the 3-year OS rate of around $70 \%$ in the two trials indicates that extended treatment with carfilzomib can produce promising results when used early in the treatment pathway. In our study in RRMM patients, carfilzomib maintenance was well tolerated, and the incidence and severity of known toxicities were similar to those in previous studies. Maintenance was associated with a higher rate of $M R D$ negativity at 6 , but not at 12 months, perhaps linked to the reduction from four to two doses per cycle of carfilzomib. Thus, patients in MUKfive trial who received $\mathrm{KCd}$ followed by carfilzomib maintenance had a combined median PFS of 18.1 months, which was slightly shorter than the median of 22.2 months for patients treated at second line in ENDEAVOR. In the A.R.R.O.W. study, ${ }^{24}$ a dose of $70 \mathrm{mg} / \mathrm{m}^{2}$ was administered weekly until progression to patients treated at third line, with the median treatment duration being 38 weeks (range, 0.1-84.1), suggesting that it may be possible to deliver a weekly maintenance schedule for an extended period.

Our results indicate potentially superior activity of $\mathrm{KCd}$ over VCd, in terms of $\geq V G P R$ rate in patients with adverse genetic risk, and ORR in those relapsing early $(<12$ months) from first-line therapy. The superior major response rate for participants with adverse risk in the $\mathrm{KCd}$ arm cannot be explained by higher discontinuation rates in the VCd arm, as the major response rates were similar in the two arms for standard-risk patients among whom the difference in discontinuation rates was more pronounced (56.7\% for VCd compared to $7.3 \%$ for $\mathrm{KCd}$ ). We also observed that patients with $\operatorname{del}(17 \mathrm{p})$ and adverse IgH translocations may have benefited especially from $\mathrm{KCd}$ in terms of VGPR (Online Supplementary Figure S4B). Despite a higher VGPR rate in adverse-risk patients in the carfilzomib arm, we did not observe a difference in MRD-negative response, nor a PFS benefit. This is likely because the short duration of triplet therapy (6 cycles), and limited carfilzomib maintenance may be insufficient for optimal disease response in these relapsed patients. We note with interest that the $\mathrm{KCd}$ regimen, when used in newly diagnosed patients ineligible for ASCT, followed by carfilzomib maintenance until progression, was recently reported to overcome the inferior prognosis of high-risk patients. However, these frontline studies used nine cycles of KCd followed by carfilzomib maintenance with four doses per cycle until progression, altogether a more intensive regimen than in the MUKfive study. ${ }^{25}$ There is, therefore, increasing evidence that carfilzomib has good activity in adverse-risk disease, although regimen intensity may be vital. In this context, it is interesting to consider the recent ENDURANCE study, ${ }^{11}$ in which carfilzomib was compared with bortezomib in NDMM patients when given in combination with lenalidomide and dexamethasone. In this trial, high-risk patients, apart from those with $\mathrm{t}(4 ; 14)$, were excluded.

One attraction of the $\mathrm{KCd}$ regimen is its relatively modest cost, hence its use in studies for RRMM and NDMM patients. In the Nordic CARFI phase II study, patients were treated at first relapse with four cycles of $\mathrm{KCd}$ followed by ASCT conditioned with high-dose melphalan plus two doses of carfilzomib. ${ }^{26} \mathrm{KCd}$ has been used to treat non-transplant-eligible, newly diagnosed patients, with the carfilzomib given bi-weekly or weekly, ${ }^{12,27}$ for up to nine cycles of induction, followed by carfilzomib maintenance. The regimen was reported to have been tolerated well in the older patient group, as we also found.

In summary, the results from the MUKfive study demonstrate the non-inferiority of carfilzomib over bortezomib in combination with cyclophosphamide and dexamethasone in the early relapse setting, and the efficacy of carfilzomib maintenance. Our data are also consistent with the high activity of carfilzomib in adverse-risk disease, both in terms of a higher VGPR rate, when compared with 
that achieved in response to bortezomib, and in the efficacy of maintenance therapy. With an increasing number of patients who, at first relapse, are exposed or refractory to bortezomib and lenalidomide, KCd followed by carfilzomib maintenance represents an economically competitive and clinically feasible regimen, reserving other novel agent combinations such as those with CD38 and pomalidomide, or the new agents targeting B-cell maturation antigen and $\mathrm{T}$-cell redirecting therapies, for a subsequent relapse or progression. Further studies are needed to explore the potential greater activity of carfilzomib in patients with adverse-risk disease, in whom the tolerability during prolonged use may make this the proteasome inhibitor of choice.

\section{Disclosures}

KLY reports grants and personal fees from Amgen, Celgene, Novartis, Janssen, and Takeda. HWA reports grants from Myeloma UK, during the conduct of the study; grants from Amgen and personal fees from Amgen, Karyopharm, Takeda, Novartis, Janssen, outside the submitted work. MFK reports personal fees from Abbvie and Amgen; grants and personal fees from Celgene; grants, personal fees and other from Janssen; and personal fees and other from Takeda, outside the submitted work. $K R$ reports grants and personal fees from Celgene, Janssen, Takeda, and Amgen; and personal fees from Abbvie, Sanofi, Oncopeptides, outside the submitted work. LF reports grants and non-financial support from Amgen, during the conduct of the study. SHa reports personal fees from Amgen, outside the submitted work. CW reports personal fees from Amgen, outside the submitted work. NKR reports personal fees from Amgen, during the conduct of the study. JCr reports personal fees from Celgene, outside the submitted work. GM reports other from AbbVie, Amgen, Celgene, Janssen, Oncopeptide, Roche, and Takeda, outside the submitted work. FD reports grants and other from Celgene and Janssen; and other from AbbVie, Adaptive Biotech, Amgen, Oncopeptide, Roche, Sanofi, and Takeda, outside the submitted work. RGO reports personal fees from Amgen, Janssen, Takeda, and Adaptive Biotech. RD (RMdT), SHi, DS, MG, JCa, CB, and $S R B$ have nothing to disclose.

\section{Contributions}

$K L Y, D S, L F, C W, J C a, R G O, G M, F D$ and $S R B$ designed the study. KLY, KR, MG, NR, CW, JCa, NKR, RGO and MFK performed the research and collected data. DS and LF performed trial and data management. HWA, SHa and CB performed independent clinical review of endpoint data. SHi and SRB performed statistical analyses. RMdT and RGO performed analysis of minimal residual disease. MFK and JCr performed central assessment of genetic risk. KLY, HWA, CB, SHi and SRB wrote the manuscript. All authors provided input into, and had control over, the final version of the manuscript.

\section{Acknowledgments}

The authors are deeply indebted to all the participants in this study and to their families and carers. We would like to thank all staff, past and present, at the participating hospitals: University College London Hospital, Leicester Royal Infirmary, Churchill Hospital Oxford, Nottingham University Hospitals, King's College Hospital, Imperial College London Healthcare NHS Trust, Royal Hallamshire Hospital, St James University Hospital, University Hospital of Wales, University Hospital Southampton NHS Foundation Trust, Birmingham Heartlands Hospital, Bristol Haematology and Oncology Centre, Manchester Royal Infirmary, Royal Bournemouth General Hospital, The Christie Hospital, Royal Devon \& Exeter Hospital, Princess Royal University Hospital, Royal Marsden Hospital, Lincoln County Hospital, New Cross Hospital, Ninewells Hospital, Pilgrim Hospital Boston, St Bartholomew's Hospital, Beatson West of Scotland Cancer Centre, University Hospital of North Tees, Queens Hospital Burton, Royal Cornwall Hospital, Royal Sussex County Hospital, Torbay District General Hospital, University Hospital Coventry, Addenbrooke's Hospital, Ayr Hospital, Countess of Chester Hospital, George Eliot Hospital, Grantham and District Hospital, Crosshouse Hospital, and Northwick Park Hospital. The authors would like to thank the Myeloma UK Clinical Trials Coordinating Office Trial Steering Committee and Data Monitoring and Ethics Committee members, without whom the trial would not have been possible: Chris Twelves, Kevin Boyd, Michael Brown, Philippe Moreau, Simon Rule, Tomasz Burzykowski, Curly Morris, Alan Anthoney, Graham Jackson, James Wason, Malcolm Ranson, Richard Soutar, and Roger Ahern. The authors are also grateful to the staff at Leeds Institute of Clinical Trials Research, who made this study possible: Lucy Bailey, Sue Bourne, Wendy Burton, Jenny Fell, Suja George, Walter Gregory, Diane Hartley, Emma Ingleson, Jessica Kendall, Paul McGarry, Rachel Naylor, Matthew Newby, Katie Robinson, Saqib Saghir, Alex Szubert, and Alan Wan.

\section{Funding}

$K L Y$ receives funding from the UCL/UCLH Biomedical Research Centre.

\section{Data-sharing statement}

Any requests for trial data will be reviewed by the trial management group in the first instance. Only requests that have a methodologically sound proposal and whose proposed use of the data has been approved by the independent trial steering committee will be considered. Proposals should be directed to the corresponding author in the first instance; to gain access; data requestors will need to sign a data access agreement. The study protocol is publicly available. ${ }^{15}$

\section{References}

1. Moreau P, San Miguel J, Sonneveld P, et al. Multiple myeloma: ESMO clinical practice guidelines for diagnosis, treatment and follow-up. Ann Oncol. 2017;28(suppl_4):iv52iv61.

2. Yong K, Gonzalez-McQuire S, Szabo Z, Schoen P, Hajek R. The start of a new wave: developments in proteasome inhibition in multiple myeloma. Eur J Haematol. 2018 Mar 30. [Epub ahead of print]
3. Bringhen S, Larocca A, Rossid D, et al Efficacy and safety of once-weekly bortezomib in multiple myeloma patients. Blood. 2010;116(23):4745-4753.

4. Dimopoulos MA, Moreau P, Palumbo A, et al. Carfilzomib and dexamethasone versus bortezomib and dexamethasone for patients with relapsed or refractory multiple myeloma (ENDEAVOR): a randomised, phase 3 open-label, multicentre study. Lancet Oncol. 2016;17(1):27-38.

5. Stewart AK, Rajkumar SV, Dimopoulos MA, et al. Carfilzomib, lenalidomide, and dexam- ethasone for relapsed multiple myeloma. $\mathrm{N}$ Engl J Med. 2015;372(2):142-152.

6. Dimopoulos M, Quach H, Mateos MV, et al. Carfilzomib, dexamethasone, and daratumumab versus carfilzomib and dexamethasone for patients with relapsed or refractory multiple myeloma (CANDOR): results from a randomised, multicentre, open-label, phase 3 study. Lancet. 2020;396(10245):186197.

7. Bringhen S, Milan A, Ferri C, et al. Cardiovascular adverse events in modern myeloma therapy - incidence and risks. A 
review from the European Myeloma Network (EMN) and Italian Society of Arterial Hypertension (SIIA). Haematologica. 2018;103(9):1422-1432.

8. Besse A, Besse L, Kraus M, et al. Proteasome inhibition in multiple myeloma: head-tohead comparison of currently available proteasome inhibitors. Cell Chem Biol. 2019;26(3):340-351.

9. Dimopoulos MA, Goldschmidt $\mathrm{H}$, Niesvizky R, et al. Carfilzomib or bortezomib in relapsed or refractory multiple myeloma (ENDEAVOR): an interim overall survival analysis of an open-label, randomised, phase 3 trial. Lancet Oncol. 2017;18(10):1327-1337.

10. Facon T, Lee JH, Moreau P, et al. Randomized phase 3 study of carfilzomib or bortezomib with melphalan-prednisone for transplant-ineligible, NDMM patients. Blood. 2019;133(18):1953-1963

11. Kumar SK, Jacobus SJ, Cohen AD, et al. Carfilzomib or bortezomib in combination with lenalidomide and dexamethasone for patients with newly diagnosed multiple myeloma without intention for immediate autologous stem-cell transplantation (ENDURANCE): a multicentre, open-label, phase 3, randomised, controlled trial. Lancet Oncol. 2020;21(10):1317-1330

12. Bringhen S, Petruccim MT, Larocca A, et al. Carfilzomib, cyclophosphamide, and dexamethasone in patients with newly diagnosed multiple myeloma: a multicenter, phase 2 study. Blood. 2014;124(1):63-69.

13. Bringhen S, D'Agostino M, De Paoli L, et al. Phase 1/2 study of weekly carfilzomib, cyclophosphamide, dexamethasone in newly diagnosed transplant-ineligible myeloma. Leukemia. 2018:32(4):979-985.

14. Reeder CB, Reece DE, Kukreti V, et al. Cyclophosphamide, bortezomib and dex- amethasone induction for newly diagnosed multiple myeloma: high response rates in a phase II clinical trial. Leukemia. 2009;23(7): 1337-1341.

15. Brown S, Hinsley S, Ballesteros M, et al. The MUK five protocol: a phase II randomised, controlled, parallel group, multi-centre trial of carfilzomib, cyclophosphamide and dexamethasone (CCD) vs. cyclophosphamide, bortezomib (Velcade) and dexamethasone (CVD) for first relapse and primary refractory multiple myeloma. BMC Hematol. 2016;16:14.

16. Rajkumar SV, Harousseau JL, Durie B, et al. Consensus recommendations for the uniform reporting of clinical trials: report of the International Myeloma Workshop Consensus Panel 1. Blood. 2011;117(18): 4691-4695.

17. Rawstron AC, Chile JA, de Tute RM, et al. Minimal residual disease assessed by multiparameter flow cytometry in multiple myeloma: impact on outcome in the Medical Research Council Myeloma IX Study. I Clin Oncol. 2013;31(20):2540-2547.

18. de Tute RM, Rawstron AC, Cairns DA, et al. Impact of minimal residual disease in transplant ineligible myeloma patients: results from the UK NCRI Myeloma XI Trial. Blood. 2016;128(22):245.

19. Shah V, SherbournenAL, Walker BA, et al. Prediction of outcome in newly diagnosed myeloma: a meta-analysis of the molecular profiles of 1905 trial patients. Leukemia. 2018;32(1):102-110

20. Fine JP, Gray RJ. A proportional hazards model for the subdistribution of a competing risk. J Am Stat Ass. 2012:496-509.

21. Robins JM, Finkelstein DM. Correcting for noncompliance and dependent censoring in an AIDS clinical trial with inverse probability of censoring weighted (IPCW) log-rank tests. Biometrics. 2000;56(3):779-788.

22. Moreau P, Dimopoulos MA, Yong K, et al. Isatuximab plus carfilzomib/dexamethasone versus carfilzomib/dexamethasone in patients with relapsed/refractory multiple myeloma: IKEMA phase III study design Future Oncol. 2020;16(2):4347-4358.

23. Moreau P, Stewart KA, Dimopoulos M, et al Once-weekly $(70 \mathrm{mg} / \mathrm{m}(2))$ vs twice-weekly $(56 \mathrm{mg} / \mathrm{m}(2))$ dosing of carfilzomib in patients with relapsed or refractory multiple myeloma: A post hoc analysis of the ENDEAVOR, A.R.R.O.W., and CHAMPION-1 trials. Cancer Med. 2020;9(9):29892996.

24. Moreau P, Mateos MV, Berenson JR, et al. Once weekly versus twice weekly carfilzomib dosing in patients with relapsed and refractory multiple myeloma (A.R.R.O.W.) interim analysis results of a randomised, phase 3 study. Lancet Oncol. 2018;19(7):953964.

25. Mina R, Bonello F, Petrucci MT, et al Carfilzomib, cyclophosphamide and dexamethasone for newly diagnosed, high-risk myeloma patients not eligible for transplant: a pooled analysis of two studies. Haematologica. 2021:106(4):1079-1085.

26. Gregersen H, Peceliunas V, Remes K, et al. A randomized phase 2 trial comparing carfilzomib-dexamethasone vs observation as maintenance after induction with carfilzomib-cyclophosphamide-dexamethasone in salvage ASCT in multiple myeloma: a trial by the Nordic Myeloma Study Group. Blood. 2019;134(Suppl_1):601.

27. Bringhen S, Mina R, Petrucci MT, et al. Once-weekly versus twice-weekly carfilzomib in patients with newly diagnosed multiple myeloma: a pooled analysis of two phase 1/2 studies. Haematologica. 2019;104 (8):1640-1647. 